Service social

\title{
À qui profitent les lois de la mission sociale?
}

\section{Paule Duchesneau et Réjean Landry}

Volume 38, numéro 2-3, 1989

Les politiques sociales

URI : https://id.erudit.org/iderudit/706438ar

DOI : https://doi.org/10.7202/706438ar

Aller au sommaire du numéro

Éditeur(s)

École de service social de l'Université Laval

ISSN

1708-1734 (numérique)

Découvrir la revue

Citer cet article

Duchesneau, P. \& Landry, R. (1989). À qui profitent les lois de la mission sociale? Service social, 38(2-3), 196-219. https://doi.org/10.7202/706438ar

\section{Résumé de l'article}

Cet article est une analyse comparative de l'action gouvernementale en matière de lois sociales adoptées par l'Assemblée nationale du Québec entre 1960 et 1985. Recherche empirique à caractère quantitatif, la démarche de l'auteur s'inspire de la théorie des biens publics, pour parler ensuite de la mission sociale de l'État et, finalement, présenter et analyser les résultats d'une étude conduite sur le terrain. 
DuChesneau, Paule, professionnelle de recherche, Groupe de recherche sur les interventions gouvernementales, Département de science politique, Université Laval.

LANDRY, Réjean, professeur au Département de science politique, Université Laval.

\section{À qui profitent les lois de la mission sociale ?*}

\section{Paule Duchesneau Réjean Landry}

Les politiques gouvernementales régissent I'allocation des ressources au sein d'une collectivité. Bien que chaque individu soit concerné par les décisions des gouvernants, certains sont davantage affectés que d'autres par l'implantation de certaines mesures gouvernementales. Les spécialistes des politiques gouvernementales se sont beaucoup intéressés aux allocations concernant les ressources budgétaires et le partage du fardeau fiscal. Plusieurs études empiriques (Mueller, 1987) remettent sérieusement en doute la thèse voulant que les allocations de ressources effectuées à travers les politiques gouvernementales engendrent un processus de redistribution des riches vers les pauvres. Une étude des dépenses de transfert du gouvernement américain démontre que les groupes les mieux organisés profitent davantage que les autres de cette forme de redistribution de ressources (Tullock, 1983). Plus près de nous, plusieurs sociologues et politologues se sont efforcés de démontrer que la transformation de l'État québécois et de ses politiques ont mieux servi les intérêts de la nouvelle classe moyenne que ceux du reste de la société (Guindon, 1964; McRoberts, 1988). Diagnostiquant I'état des services de santé et des services sociaux, le rapport Rochon en arrivait à conclure que le système de production de soins de santé 
et de services sociaux était pris en otage par les groupes de producteurs les mieux organisés. Par ailleurs, les rapports des Commissions d'enquête sur les services publics, particulièrement dans le secteur de la santé et des services sociaux, laissent croire que l'amélioration des services gouvernementaux passe obligatoirement par de grandes réformes institutionnelles (Rapport CastonguayNepveu et Rapport Rochon).

Ces interprétations et les travaux empiriques qui en dérivent arrivent donc tous à la même conclusion : le transfert de richesse effectué à travers les politiques publiques profite davantage à certains groupes qu'à d'autres. À qui donc profitent les interventions de l'État ? Cette question difficile et controversée sera abordée à travers l'analyse des lois sociales adoptées par l'Assemblée nationale du Québec entre 1960 et 1985. L'analyse nous conduira à distinguer l'action des gouvernements qui se sont succédés de 1960 à 1985 en procédant à l'analyse comparative : 1) du nombre de lois et d'interventions adoptées; 2) des types de lois adoptées; 3) du nombre d'interventions par type de lois; 4) des domaines d'intervention; 5) des cibles d'intervention; et 6) des types de biens offerts. Ce qui est ici inféré, relativement à la fréquence des observations, tient davantage à l'intérêt porté à un objet donné, d'un gouvernement à l'autre. II va sans dire que l'approche comparative ne concerne nullement l'impact social réel de l'intervention mais davantage le fait d'intervenir d'une façon plutôt que d'une autre. Avant d'entrer de plein pied dans l'univers des données empiriques, il importe d'identifier les conditions inhérentes au processus d'élaboration des politiques qui sont susceptibles d'engendrer des biais dans le transfert de richesse. Au préalable, il s'impose toutefois de justifier sommairement l'impact des lois sur le transfert de richesse.

La distribution des revenus entre les membres d'une collectivité ne dépend pas seulement des dépenses budgétaires et des mesures fiscales. Les gouvernements peuvent tout autant affecter les revenus des particuliers et les prix des biens et services au moyen de l'adoption de lois et de règlements. Ainsi, les lois relatives aux pratiques professionnelles, à la sécurité et à la qualité des biens de consommation, aux contrats et à la propriété déterminent, à des degrés divers, la distribution des revenus privés des particuliers. Les lois affectent aussi les bénéfices non monétaires (bénéfices symboliques ou axiologiques) des individus comme en témoignent les mesures gouvernementales relatives à la promotion de la langue française ou la protection de la qualité de l'environnement. En fin de compte, les lois qui ont pour effet d'entraîner des modifications de prix du marché de certains biens et services ne font rien d'autre que de changer la distribution des revenus nets réels. 
L'impact des lois sur le transfert de richesse est probablement plus difficile à évaluer que les effets des dépenses budgétaires et des impôts. Cette difficile tâche s'impose pourtant avec force, d'autant plus que les lois cristallisent plus solidement les bénéfices que les dépenses budgétaires. Pour les bénéficiaires de l'action de l'État, les bénéfices qui se concrétisent à travers les lois peuvent s'avérer préférables à des gains monétaires émergeant du budget public puisque des bénéfices découlant des lois durent plus longtemps que des bénéfices monétaires qui sont remis en cause chaque année au moment de la préparation du budget de l'État. Mais ces bénéfices engendrés par les transferts de richesse profitent-ils également à tous les membres de la collectivité ?

\section{Quelques concepts de la théorie des biens publics}

L'adoption de lois affecte réellement ou potentiellement les intérêts des individus en procédant à I'allocation autoritaire des ressources au sein des membres d'une collectivité. Dans un monde d'information parfaite où les coûts d'organisation de l'action collective sont nuls et les décisions adoptées en suivant la règle de I'unanimité, l'allocation des ressources engendre des résultats optima de Pareto : I'allocation des ressources entre les alternatives de décision est telle que personne ne pourrait améliorer son sort sans affecter négativement celui de quelqu'un d'autre. L'efficience de Pareto s'avère toutefois difficile à atteindre en raison du caractère irréaliste des conditions à satisfaire. Ainsi, l'information à la disposition des législateurs n'est jamais parfaite. Ceux-ci ne connaissent parfaitement, ni les attentes des particuliers, ni les effets des alternatives envisagées. Les inconvénients qui en résultent ne peuvent être atténués que par des investissements dans la cueillette et l'analyse d'information. La demande d'information des législateurs incite certains groupes d'individus à s'engager dans l'offre d'information. On assiste donc à la création d'une sorte de marché sur lequel se transigent des renseignements au sujet des alternatives de politiques publiques et des effets qui en découlent. Cependant, dans un monde où l'information et l'organisation d'actions collectives sont coûteuses, certains groupes d'individus parviendront à recueillir l'information requise et à organiser leurs activités de démarchage à des coûts inférieurs à d'autres. Ces différences de coûts sont assimilables à des coûts de démarrage (McCormick et Tollison, 1981) : les groupes qui ont déjà assumé leurs coûts de démarrage pour atteindre des objectifs étrangers aux activités de démarchage auront une incitation plus élevée que les autres à investir dans l'acquisition d'information et l'organisation d'activités de démarchage auprès des législateurs pour 
obtenir le résultat qui profiterait le plus aux intérêts de leurs membres. En d'autres mots, pour certains groupes, la réalisation d'activités de démarchage constitue un sous-produit financé à travers l'accomplissement d'autres activités.

Olson $(1965 ; 1982)$ propose une façon originale de distinguer les groupes selon le niveau des coûts de démarrage qu'ils doivent supporter. Ainsi, les individus concernés par les lois à titre de consommateurs et de contribuables sont peu incités à assumer les coûts d'organisation qu'exigerait la présentation de leurs demandes aux législateurs parce que ces coûts tendent à excéder les bénéfices qu'ils peuvent espérer obtenir en réussissant à imposer leurs vues. En règle générale, les coûts d'organisation tendent à excéder les bénéfices parce que les taxes supportées par les contribuables et les dépenses effectuées par les consommateurs se répartiront sur un large éventail d'items dont chacun ne constitue généralement qu'une fraction infime. En conséquence, les groupes composés de contribuables et de consommateurs constituent des coalitions à coûts élevés. Inversement, les individus concernés à titre de détenteurs de facteurs de production sont fortement incités à assumer les coûts d'organisation requis pour la présentation de leurs demandes aux législateurs parce que les lois qui concernent leurs facteurs de production affectent de façon très significative leurs revenus. II en va ainsi parce que les détenteurs de facteurs de production tirent l'essentiel de leurs revenus de la possession d'un seul facteur de production. Les groupes composés de détenteurs de facteurs de production représentent donc des coalitions à faibles coûts. Ces différences expliquent pourquoi les groupes de détenteurs de facteurs de production sont plus incités à investir des ressources dans des activités de démarchage que les groupes de consommateurs et de contribuables qui sont, quant à eux, confrontés au problème du resquillage. Si bien que les législateurs reçoivent le point de vue des coalitions à faibles coûts tandis que les coalitions à coûts élevés demeurent ignorantes des coûts et bénéfices, voire de l'existence même des projets de loi.

Ce premier biais se paire à une structure d'incitation qui récompense les groupes et les législateurs qui s'engagent dans le transfert de richesse. Les groupes d'intérêt peuvent en effet maximiser les bénéfices de leurs membres en travaillant à l'accroissement de la richesse de l'ensemble de la société, ou en tentant d'obtenir une part plus importante de la richesse existante. Mais un groupe qui œuvrerait à l'augmentation de la richesse collective produirait en fait un bien public dont il supporterait la totalité des coûts en échange de maigres bénéfices pour les membres qui le composent. D'après Olson (1982: 44) aucun groupe n'est incité à faire des sacrifices significatifs dans l'intérêt de l'ensemble de la société. Au contraire, 
prétend-t-il, les groupes qui veulent le mieux servir leurs membres y arrivent en tentant de s'approprier une part relative plus importante de la richesse de l'ensemble de la société. Si bien que l'action des groupes d'intérêt est davantage orientée vers le transfert que vers l'augmentation de la richesse. Les groupes d'intérêt sont donc incités à resquiller au détriment de l'ensemble de la société.

De la même manière, les législateurs n'ont pas intérêt à adopter des mesures législatives favorisant des coalitions à coûts élevés puisque celles-ci sont confrontées au problème du resquillage. Les législateurs sont incités à voter plus souvent des lois qui profitent aux coalitions à faibles coûts qu'aux coalitions à coûts élevés. De plus, les législateurs sont davantage incités à faire supporter les coûts des interventions favorisant les coalitions à faibles coûts par les membres des coalitions à coûts élevés que de faire supporter les coûts des bénéfices allant aux détenteurs de facteurs de production par les consommateurs et les contribuables. Or, les bénéfices fournis aux coalitions à faibles coûts tendent à s'apparenter à des biens privés en raison du critère d'exclusion qui régit la consommation de bénéfices réservés à des catégories particulières de détenteurs de facteurs de production. Les législateurs sont donc incités à produire davantage des biens privés que des biens publics.

Cette structure d'incitation prend appui sur une motivation fondamentale des législateurs et des leaders de groupes : le désir d'être réélu. Or, les représentants qui veulent être réélus à la tête de leur parti ou de leur groupe cloivent servir les intérêts de leur base électorale. Cet objectif les incite à favoriser les mesures législatives qui maximisent leur probabilité de réélection. D'ailleurs, un représentant qui n'agit pas de façon à maximiser les votes sera facilement défait par un opposant qui cherche à maximiser les votes.

En résumé, les coûts d'information et d'organisation inhérents au fonctionnement des processus politiques engendrent le transfert de richesse, et la motivation des législateurs et des groupes d'intérêt crée un biais qui avantage les groupes de détenteurs de facteurs de production au détriment des groupes de consommateurs et de contribuables (Downs, 1957; Olson, 1965, 1982; Moe, 1980, 1981; Hayes, 1981; Frey, 1983).

En théorie, les coûts d'information et les coûts d'organisation de l'action collective détermineraient donc des lois régressives. Les évidences empiriques à l'appui de cette thèse ne sont pas faciles à trouver. À la suite d'un examen systématique des études sur I'incidence des dépenses gouvernementales et du partage du fardeau fiscal au Canada, Dahlby (1986) conclut que nous sommes encore loin de savoir qui profite des dépenses publiques et qui assume le coût des impôts. Les principales difficultés à la base de ces études 
empiriques concernent l'impartition aux individus des bénéfices résultant des biens produits par l'État et le partage des coûts entre les différentes catégories de groupes de particuliers. La thèse de la régressivité des mesures gouvernementales s'appuie néanmoins sur quelques confirmations empiriques. Ainsi, Lemelin (1980:71) a montré que les individus les moins scolarisés subventionnent le coût des études universitaires des enfants des familles de classe moyenne. D'autre part, Payette et Vaillancourt (1986) ont démontré que les impôts du gouvernement du Québec sont légèrement régressifs mais que les dépenses de l'État bénéficient davantage aux ménages à faible revenu qu'aux autres. À notre connaissance, personne ne s'est encore interrogé sur le caractère plus ou moins régressif des lois - à l'exception des lois fiscales. Nous avons voulu nous attaquer à cette question à travers l'étude des lois de la mission sociale du gouvernement du Québec.

\section{Les missions de l'État et la mission sociale}

Les interventions gouvernementales extraites des lois ont été classées suivant les catégories du système Plan Programme Budget (PPB) du gouvernement du Québec. Ce plan distingue les activités de l'État selon quatre grandes missions qui sont : la mission gouvernementale et administrative; la mission économique; la mission sociale; la mission éducative et culturelle. Les composantes de chacune de ces missions sont les domaines d'intervention; on en dénombre 58. La mission sociale comprend 11 domaines d'intervention qui possèdent chacun de 6 à 10 indicateurs. Ces domaines sont : 1) la sécurité sociale; 2) la santé et les services sociaux; 3) la famille; 4) la condition féminine; 5) les relations de travail; 6) les conditions de travail; 7) l'aménagement social; 8) la consommation; 9) l'immigration; 10) l'émigration; 11) les affaires religieuses.

\section{Technique de collecte des données}

La phase de la cueillette des données s'est subdivisée en quatre étapes principales : la détermination de l'unité d'analyse; la sélection des documents à analyser; l'enregistrement des données; la vérification des données.

L'analyse comparative des lois s'avère hasardeuse car celles-ci constituent un instrument verbal d'intervention qui se prête plus difficilement à la quantification que d'autres formes de ressources. Toutefois, le fait que le contenu de chaque loi soit spécifique n'implique pas qu'il y ait absence de régularités. La recherche de régularités passe par l'identification d'éléments communs permettant 
de comparer les lois les unes aux autres. La proposition d'intervention gouvernementale offre davantage cette garantie que d'autres unités d'analyse telles que la loi, l'article de loi, ou le thème de la loi. Une proposition d'intervention gouvernementale se compose de quatre éléments :

- Qui énonce l'intervention ? ex.: le ministre des Affaires sociales.

- Quel est le verbe connecteur utilisé dans l'intervention ? ex. : pourra autoriser.

- Quel est l'attribut de l'intervention votée ? ex. : à bénéficier du régime québécois d'assurance-maladie, pour au plus quatre mois.

- Quels sont les groupes d'individus ou les institutions visés par l'intervention ? ex. : une personne qui quitte le Québec pour une autre province où un tel régime n'existe pas.

L'Assemblée nationale du Québec a adopté 1662 lois publiques au cours de la période 1960-1985. De ce nombre, 487 lois concernent la mission sociale. L'ampleur de la tâche à accomplir était donc considérable. Aussi, avons-nous choisi d'étudier les résumés des lois plutôt que les textes de lois dans leur entièreté. Ce corpus est d'autant plus accessible que l'Assemblée nationale produit des recueils annuels des résumés des lois. Le résumé moyen totalise une trentaine de lignes de texte.

L'enregistrement des données a été réalisé à l'aide d'un questionnaire fermé. La technique d'analyse de contenu utilisée peut être assimilée à une simulation d'entrevues : des analystes posent des questions à des documents et répondent à un questionnaire en utilisant des consignes concernant les aspects conceptuels et techniques du questionnaire. La première tâche des analystes consistait à identifier chacune des propositions d'intervention contenues dans le résumé de la loi et à l'inscrire sur le questionnaire. La seconde étape concernait l'analyse proprement dite de la proposition d'intervention. Cette tâche était facilitée par l'utilisation du questionnaire fermé qui comprenait des questions relatives aux attributs des interventions, aux caractéristiques des coûts et des bénéfices des interventions, de même qu'aux destinataires des coûts et des bénéfices. L'essentiel de ces questions s'inspire de concepts provenant de la théorie économique des biens publics. D'ailleurs, l'utilisation de questions concernant les destinataires des interventions résout l'une des principales difficultés des études sur les biens publics relativement à l'impartition des bénéfices et des coûts des mesures gouvernementales.

Le questionnaire a été administré par trois professionnels de recherche travaillant à temps complet à la réalisation du projet. Les 
questionnaires remplis ont été vérifiés manuellement par une quatrième personne dans le but d'accroître la fiabilité et la validité de I'analyse. Les réponses des questionnaires vérifiés ont par la suite été enregistrées sur ruban d'ordinateur. La validité de l'analyse fut alors à nouveau vérifiée à l'aide d'un programme informatique dont le but était de détecter les réponses illogiques. Ces inconsistances logiques ont été vérifiées manuellement. Ces opérations de contrôle de qualité concernant la fiabilité et la validité des données nous ont amenés à corriger un peu moins de $5 \%$ des questionnaires remplis.

\section{Présentation des résultats Du global vers le particulier}

De 1960 à 1985, l'Assemblée nationale du Québec a adopté 487 lois concernant la mission sociale de l'État. Les gouvernements ont donc en moyenne adopté un peu moins de 19 lois sociales par année. Contrairement à toute attente, le nombre annuel moyen de lois adoptées au cours des années 60 est inférieur aux périodes subséquentes. La période durant laquelle la révolution tranquille battait son plein, soit entre 1962 et 1966, a engendré un score moyen un peu inférieur à 14 lois par année, soit le score moyen le plus bas pour la période allant de 1960 à 1985. À nouveau contre toute attente, les gouvernements les plus actifs dans la mission sociale au cours des années 70 et 80 ne sont pas les deux gouvernements péquistes (PQ) mais les gouvernements libéraux (PLQ) de 70-73 et 73-76 dont le nombre annuel moyen de lois votées atteint 32,3 et 20,6 respectivement (Tableau 1).

\section{TABLEAU 1}

Lois de la mission sociale par gouvernement, 1960-1985

\begin{tabular}{|l|c|c|}
\hline Gouvernement & nombre de lois & moyenne/an \\
\hline & 38 & \\
PLQ 60-62 & 55 & 19 \\
PLQ 62-66 & 66 & 13,7 \\
UN 66-70 & 97 & 16,5 \\
PLQ 70-73 & 62 & 32,3 \\
PLQ 73-76 & 93 & 20,6 \\
PQ 76-81 & 75 & 18,6 \\
PQ 81-85 & 487 & 18,7 \\
TOTAL & & 18,7 \\
\hline
\end{tabular}


D'autre part, les gouvernements réalisent leurs interventions législatives par l'introduction de modifications aux lois existantes six fois sur dix, alors qu'ils recourent à l'adoption de lois nouvelles près de quatre fois sur dix. L'abrogation directe de lois constitue une mesure exceptionnelle : les gouvernements y recourent moins de un demi de un pourcent du temps. Un si faible taux d'abrogation confirme I'hypothèse de Rose (1983 : 76) voulant qu'une fois adoptée, chaque loi a ses supporteurs à l'intérieur du gouvernement et ses bénéficiaires à l'extérieur du gouvernement.

Le gouvernement de l'Union nationale (UN) 66-70 a adopté près de $55 \%$ de lois nouvelles alors que les gouvernements libéraux de la révolution tranquille, PLQ 60-62 et PLQ 62-66, recouraient chacun à moins de $30 \%$ de lois nouvelles pour réaliser leurs interventions. II en va de même pour la période subséquente où, contre toute attente, on observe que le gouvernement libéral de 1970-1973 a beaucoup plus recouru à l'adoption de lois nouvelles que le premier gouvernement péquiste. En effet, les gouvernements libéraux de la révolution tranquille et le premier gouvernement péquiste, auxquels on associe généralement de grands projets collectifs et des changements sociaux importants, ont, en fin de compte, recouru plus souvent que les autres à la modification des lois existantes (Tableau 2). Ces résultats infirment le postulat qui suppose que les grands changements passent par l'adoption des lois nouvelles plutôt que par la modification des lois en vigueur. Ces résultats doivent toutefois être interprétés de façon pruderite puisqu'il n'existe probablement pas une association parfaite entre l'ampleur des changements découlant des lois et la proportion de lois nouvelles adoptées.

L'examen du nombre de propositions selon les types de lois adoptées donne une image assez semblable (Tableau 3). On retrouve en effet un peu moins de quatre propositions sur dix dans les lois nouvelles et un peu plus de six sur dix dans les lois modifiées. On peut cependant noter que si le gouvernement UN 66-70 était le champion des lois nouvelles, celles adoptées sous le gouvernement PLQ 70-73 véhiculent un plus grand nombre de propositions d'intervention gouvernementale que les autres gouvernements.

L'univers des lois de la mission sociale recouvre plusieurs domaines d'intervention qu'on peut regrouper autour de trois dimensions principales : la première concerne la santé et les services sociaux, et elle inclut, en plus du domaine santé et services sociaux, le domaine de la sécurité sociale, celui de la famille, et enfin, celui de la condition féminine. Cette première dimension regroupe 155 lois $(31,8 \%)$ et 796 propositions $(30,5 \%)$. La seconde dimension, celle du travail, qui comprend le domaine des relations de travail et celui des conditions de travail, draine 213 lois $(43,7 \%$ ) pour un total de 1274 
TABLEAU 2

Type de lois adoptées par gouvernement de 1960 à 1985

\begin{tabular}{|c|c|c|c|c|c|c|c|c|c|}
\hline \multirow[b]{2}{*}{ Type de lois } & & \multicolumn{8}{|c|}{ Gouvernement } \\
\hline & & $\begin{array}{c}\text { PLQ } \\
60-62 \\
\end{array}$ & $\begin{array}{c}\text { PLQ } \\
62-66\end{array}$ & $\begin{array}{c}\text { UN } \\
66-70\end{array}$ & $\begin{array}{c}\text { PLQ } \\
70-73\end{array}$ & $\begin{array}{c}\text { PLQ } \\
73-76\end{array}$ & $\begin{array}{c}P Q \\
76-81\end{array}$ & $\begin{array}{c}P Q \\
81-85\end{array}$ & $\begin{array}{l}\text { Total } \\
60-85\end{array}$ \\
\hline Nouvelles & $\begin{array}{l}\% \\
\mathrm{~N}\end{array}$ & 28,9 & 27,3 & 54,5 & 47,4 & 32,3 & 26,6 & 44,0 & $\begin{array}{c}38,2 \\
(186)\end{array}$ \\
\hline Modifiées & $\begin{array}{l}\% \\
N\end{array}$ & 71,0 & 72,7 & 45,4 & 51,5 & 66,1 & 73,4 & 56,0 & $\begin{array}{c}61,4 \\
(299)\end{array}$ \\
\hline Abrogées & $\begin{array}{l}\% \\
N\end{array}$ & - & - & - & 1,0 & 1,6 & - & - & $\begin{array}{c}0,004 \\
(2)\end{array}$ \\
\hline TOTAL & $N$ & 38 & 55 & 66 & 97 & 62 & 94 & 75 & 487 \\
\hline
\end{tabular}


TABLEAU 3

Nombre de propositions par type de lois selon les gouvernements, 1960-1985

\begin{tabular}{|c|c|c|c|c|c|c|c|c|c|}
\hline \multirow{2}{*}{\multicolumn{2}{|c|}{$\begin{array}{l}\text { Propositions par } \\
\text { type de lois }\end{array}$}} & \multicolumn{8}{|c|}{ Gouvernement } \\
\hline & & $\begin{array}{c}\text { PLQ } \\
60-62\end{array}$ & $\begin{array}{c}\text { PLQ } \\
62-66\end{array}$ & $\begin{array}{c}\text { UN } \\
66-70\end{array}$ & $\begin{array}{c}\text { PLQ } \\
70-73\end{array}$ & $\begin{array}{c}\text { PLQ } \\
73-76\end{array}$ & $\begin{array}{c}\text { PQ } \\
76-81\end{array}$ & $\begin{array}{c}\text { PQ } \\
81-85\end{array}$ & $\begin{array}{l}\text { Total } \\
60-85\end{array}$ \\
\hline Lois nouvelles & $\begin{array}{l}\% \\
\mathrm{~N}\end{array}$ & 23,1 & 27,3 & 47,5 & 60,9 & 21,9 & 21,1 & 37,2 & $\begin{array}{c}37,4 \\
(1019)\end{array}$ \\
\hline Lois modifiées & $\begin{array}{l}\% \\
\mathrm{~N}\end{array}$ & 76,9 & 72,7 & 52,5 & 38,9 & 77,8 & 78,8 & 62,8 & $\begin{array}{r}62,5 \\
(1701)\end{array}$ \\
\hline Lois abrogées & $\begin{array}{l}\% \\
N\end{array}$ & - & - & 0,1 & 0,3 & - & - & - & $\begin{array}{l}0,1 \\
(2)\end{array}$ \\
\hline TOTAL & $\mathrm{N}$ & 121 & 242 & 356 & 655 & 379 & 539 & 430 & 2722 \\
\hline
\end{tabular}


interventions $(48,8 \%)$. Finalement, la dimension de l'aménagement social regroupe les domaines de l'aménagement social (urbanisation, zonage, organisation du territoire), de la consommation, des services relatifs à l'immigration, ainsi que le domaine des affaires religieuses. Cette troisième dimension fait l'objet de 119 lois $(24,2 \%)$ et de 540 interventions $(20,7 \%)$.

Cette décomposition des lois de la mission sociale en dimensions et en domaines suggère que l'activité législative des gouvernements concerne non seulement les caractéristiques des bénéfices et des coûts des interventions de l'État mais aussi l'établissement de normes régissant l'accès aux bénéfices des interventions, les relations entre les groupes et l'appareil étatique, puis finalement, les règles régissant la production des services publics. Aussi importe-t-il de se demander si la législation de la mission sociale concerne la modification d'arrangements institutionnels ou de changements dans les services offerts aux individus.

Près du quart de la législation concerne les aspects institutionnels de la mission sociale. Un premier bloc de lois touche directement la création, la modification, l'abolition de structures institutionnelles, ou encore, la modification des règles de fonctionnement de structures institutionnelles. Ces interventions sur les structures institutionnelles impliquent $280(10,4 \%)$ des 2722 mesures de la mission sociale. Un second bloc de lois affecte les règles définissant les relations et les conditions de travail au sein des structures institutionnelles de l'État. L'activité législative qui en découle se manifeste à 452 reprises, soit $16,6 \%$ des propositions des lois de la mission sociale. Aussi les interventions concernant les arrangements institutionnels de l'État s'accaparent-elles $26,9 \%$ des propositions de la mission sociale (Tableau 4).

Les individus sont visés par les interventions législatives de la mission sociale à titre de détenteurs de facteurs de production du secteur privé ainsi qu'à titre de consommateurs de services publics et privés. Près de $70 \%$ des interventions s'adressent explicitement aux individus. Par ailleurs, les mesures législatives ne fournissaient pas suffisamment $d^{\prime}$ informations explicites pour identifier leur type de destinataire pour un plus de $3 \%$ des observations.

Les cibles des interventions ne varient pas énormément d'un parti gouvernemental à l'autre. Il est donc difficile de conclure qu'un parti recourt plus qu'un autre à la modification des arrangements institutionnels du secteur public. Par contre, comment peut-on expliquer que le gouvernement PLQ 60-62 et le gouvernement PQ 81-85 aient recouru relativement plus souvent que les autres à des mesures relatives aux arrangements institutionnels alors que le gouvernement PLQ 70-73 a beaucoup moins utilisé cette forme d'intervention ? Le 


\section{TABLEAU 4}

Distribution des propositions de la mission sociale selon les types de cibles et les gouvernements, 1960-1985

\begin{tabular}{|c|c|c|c|c|c|c|c|c|c|}
\hline \multirow[b]{2}{*}{ Destinataires } & & \multicolumn{8}{|c|}{ Gouvernement } \\
\hline & & $\begin{array}{c}\text { PLQ } \\
60-62\end{array}$ & $\begin{array}{c}\text { PLQ } \\
62-66\end{array}$ & $\begin{array}{c}\text { UN } \\
66-70\end{array}$ & $\begin{array}{c}\text { PLQ } \\
70-73\end{array}$ & $\begin{array}{c}\text { PLQ } \\
73-76\end{array}$ & $\begin{array}{c}\mathrm{PQ} \\
76-81\end{array}$ & $\begin{array}{c}P Q \\
81-85\end{array}$ & $\begin{array}{l}\text { Total } \\
60-85\end{array}$ \\
\hline $\begin{array}{l}\text { Arrangements } \\
\text { institutionnels }\end{array}$ & $\begin{array}{l}\% \\
N\end{array}$ & 35,6 & 23,1 & 29,6 & 18,5 & 26,1 & 29,8 & 34,2 & $\begin{array}{r}26,9 \\
(732)\end{array}$ \\
\hline Individus & $\begin{array}{l}\% \\
N\end{array}$ & 63,6 & 71,1 & 64,8 & 78,5 & 72,0 & 67,5 & 63,7 & $\begin{array}{r}69,9 \\
(1904)\end{array}$ \\
\hline Indéfinis & $\%$ & 0,8 & 5,8 & 5,6 & 3,0 & 1,9 & 2,6 & 2,1 & $\begin{array}{c}3,2 \\
(86)\end{array}$ \\
\hline TOTAL & $\mathrm{N}$ & 121 & 242 & 355 & 655 & 379 & 539 & 430 & 2722 \\
\hline
\end{tabular}


début des années 60 marquait-il une phase de construction des institutions alors que la période plus récente représenterait une phase de régulation des structures institutionnelles existantes? D'autre part, la proportion des interventions qui s'adressent aux individus évolue en dents de scie, quasi indépendamment des partis au pouvoir. Les gouvernements libéraux des périodes 70-73 et 73-76 ont été les plus généreux à l'endroit des individus alors que le premier gouvernement Lesage de 60-62 et le premier gouvernement péquiste de 76-81 ont voté la plus faible proportion de mesures destinées explicitement aux individus. Ces observations ne permettent pas $\mathrm{d}^{\prime}$ 'identifier des phénomènes de transfert de richesse, pas plus que les liens de dépendance pouvant exister entre le transfert de richesse et l'idéologie des partis gouvernementaux.

\section{Biens publics, biens privés}

Dans leurs efforts pour déterminer les bénéficiaires des mesures gouvernementales, les économistes ont développé le concept de bien public qu'ils opposent à celui de bien privé. Un bien privé est parfaitement divisible et comporte des bénéfices susceptibles d'exclusion. Un bénéfice est considéré divisible si on peut en établir la somme et la fractionner entre les récipiendaires potentiels. Des bénéfices sont jugés indivisibles dans tous les autres cas. D'autre part, un bénéfice est défini comme susceptible d'exclusion lorsque son récipiendaire potentiel doit satisfaire un critère d'éligibilité ou assumer des frais avant de pouvoir le consommer. Les bénéfices sont considérés difficiles d'exclusion dans tous les autres cas. Réciproquement, un bien public comporte des bénéfices indivisibles et difficiles d'exclusion.

Ces distinctions conceptuelles génèrent deux types de biens mixtes : les biens de clubs comportant des bénéfices indivisibles pour lesquels l'exclusion est possible et des biens communs véhiculant des bénéfices divisibles mais non susceptibles d'exclusion. Finalement, le travail sur le terrain nous a amenés à concevoir un cinquième type de biens, étiqueté biens indéfinis, pour répondre aux situations où la description des interventions législatives ne comporte pas suffisamment d'informations sur la divisibilité et l'exclusion. Le tableau 5 montre que les interventions législatives de la période 60-85 ont produit seulement $11,5 \%$ de biens publics, comparativement à $4,7 \%$ de biens privés et près de $80 \%$ de biens de clubs. Ces résultats sont congruents avec ceux d'une étude récente de Montmarquette (1988) qui a observé que la production de biens publics concernait $15 \%$ des dépenses du gouvernement canadien. Force est donc de conclure que les interventions législatives produisant des bénéfices s'adressant 
TABLEAU 5

Types de biens offerts selon les gouvernements 1960-1985

\begin{tabular}{|c|c|c|c|c|c|c|c|c|c|}
\hline \multirow{2}{*}{\multicolumn{2}{|c|}{ Types de biens }} & \multicolumn{8}{|c|}{ Gouvernement } \\
\hline & & $\begin{array}{l}\text { PLQ } \\
60-62\end{array}$ & $\begin{array}{l}\text { PLQ } \\
62-66\end{array}$ & $\begin{array}{c}\text { UN } \\
66-70\end{array}$ & $\begin{array}{l}\text { PLQ } \\
70-73\end{array}$ & $\begin{array}{l}\text { PLQ } \\
73-76\end{array}$ & $\begin{array}{c}\mathrm{PQ} \\
76-81\end{array}$ & $\begin{array}{c}\text { PQ } \\
81-85\end{array}$ & $\begin{array}{l}\text { Total } \\
60-85\end{array}$ \\
\hline Biens privés & $\%$ & 7,0 & 2,1 & 5,9 & 6,8 & 4,4 & 3,7 & 3,1 & $\begin{array}{r}4,7 \\
(124)\end{array}$ \\
\hline Biens de Clubs & $\%$ & 80,0 & 90,3 & 82,7 & 79,5 & 76,0 & 79,1 & 76,6 & $\begin{array}{r}79,9 \\
(2085)\end{array}$ \\
\hline Biens communs & $\%$ & - & 1,7 & 1,8 & 1,6 & 1,1 & 0,8 & 2,4 & $\begin{array}{l}1,5 \\
(38)\end{array}$ \\
\hline Biens publics & $\%$ & 7,8 & 3,4 & 7,6 & 10,8 & 16,8 & 14,1 & 13,7 & $\begin{array}{r}11,5 \\
(301)\end{array}$ \\
\hline Biens indéfinis & $\%$ & 5,2 & 2,5 & 2,1 & 1,3 & 1,7 & 2,3 & 4,1 & $\begin{array}{c}2,4 \\
(62)\end{array}$ \\
\hline TOTAL & $\begin{array}{l}\mathrm{N} \\
\%\end{array}$ & 115 & 237 & 341 & 628 & 363 & 511 & 415 & $\begin{array}{l}(2610)^{*} \\
100,0 \%\end{array}$ \\
\hline
\end{tabular}

* Les arrangements de production sans destinataires sont exclus. 
à tous sans restriction concernent à peine plus de $10 \%$ de l'activité législative de la mission sociale. Ce seuil passe de $11,5 \%$ à près de $14 \%$ si I'on fait l'hypothèse que les biens indéfinis sont accessibles à tous faute de critère d'exclusion. Le fait que près de $85 \%$ des biens produits $(79,9 \%$ de biens de clubs et $4,7 \%$ de biens privés) comportent des critères d'exclusion signifie que les interventions législatives produisent des biens dont la consommation est réservée à des groupes particuliers. Cette situation n'a pas vraiment changé au cours de la période étudiée si l'on excepte que la production de biens de clubs a été plus forte au cours des années 60 que par la suite, et que la production de biens publics atteint son pic sous le gouvernement libéral de la période 73-76.

Quels sont donc au juste les bénéficiaires des biens dont la consommation est régie par des critères explicites d'exclusion ?

Selon la théorie marxiste et la théorie économique classique, les bénéficiaires des mesures de l'État peuvent être catégorisés en fonction de leurs rôles dans le système de production. À cet égard, il importe de distinguer les détenteurs de facteurs de production des consommateurs, catégorie qui peut être fractionnée en trois groupes : entrepreneurs, travailleurs et détenteurs d'autres facteurs de production. Les interventions concernant les travailleurs du secteur public seront maintenant comptabilisées avec les travailleurs du secteur privé dans la catégorie travailleurs au lieu d'être identifiées à des mesures relatives aux arrangements institutionnels. Le tableau 6 rend compte des résultats découlant de ces distinctions conceptuelles. Notons tout de suite qu'il est impossible de préciser quels sont les destinataires des interventions pour près de $10 \%$ des observations (catégorie indéfinis). Les consommateurs de services publics et privés sont la cible d'un peu plus du quart de la législation de la mission sociale, les travailleurs en constituent les bénéficiaires dans $41 \%$ des cas et les entrepreneurs en profitent près de $13 \%$ du temps. Au total, $62,6 \%$ des interventions de la mission sociale procurent des bénéfices aux détenteurs de facteurs de production, comparativement à $27,7 \%$ pour les consommateurs. On s'attendait à ce que les interventions de la mission sociale visent plus souvent les consommateurs que les détenteurs de facteurs de production. La législation de la mission sociale concerne donc beaucoup plus la gestion des rapports entre les groupes de détenteurs de facteurs de production (entrepreneurs versus travailleurs dans le rapport patron-employé) que des caractéristiques des bénéfices fournis aux consommateurs de services publics et privés.

Les données du tableau 6 montrent que cette tendance de fond n'est pas beaucoup affectée par des changements de gouvernement. La performance du premier gouvernement Bourassa, qui a produit 
TABLEAU 6

Destinataires des interventions législatives selon les gouvernements, 1960-1985

\begin{tabular}{|c|c|c|c|c|c|c|c|c|c|}
\hline \multirow{2}{*}{\multicolumn{2}{|c|}{ Destinataires }} & \multicolumn{8}{|c|}{ Gouvernement } \\
\hline & & $\begin{array}{l}\text { PLQ } \\
60-62\end{array}$ & $\begin{array}{l}\text { PLQ } \\
62-66\end{array}$ & $\begin{array}{l}\text { UN } \\
66-70\end{array}$ & $\begin{array}{l}\text { PLQ } \\
70-73\end{array}$ & $\begin{array}{l}\text { PLQ } \\
73-76\end{array}$ & $\begin{array}{c}P Q \\
76-81\end{array}$ & $\begin{array}{c}\text { PQ } \\
81-85\end{array}$ & $\begin{array}{l}\text { Total } \\
60-85\end{array}$ \\
\hline Entrepreneurs & $\%$ & 4,4 & 27 & 7,9 & 11,9 & 13,2 & 16,2 & 8,4 & $\begin{array}{r}12,9 \\
(337)\end{array}$ \\
\hline Travailleurs & $\begin{array}{l}\% \\
\text { N }\end{array}$ & 40,9 & 37,5 & 40,2 & 45,2 & 34,7 & 41,9 & 44,1 & $\begin{array}{r}41,4 \\
(1080)\end{array}$ \\
\hline $\begin{array}{l}\text { Autres } \\
\text { producteurs }\end{array}$ & $\begin{array}{l}\% \\
N\end{array}$ & 12,2 & 5,1 & 12,1 & 7,0 & 10,2 & 6,7 & 8,2 & $\begin{array}{r}8,3 \\
(216)\end{array}$ \\
\hline Consommateurs & $\begin{array}{l}\% \\
N\end{array}$ & 32,1 & 19,8 & 28,7 & 26,6 & 33,3 & 25,8 & 29,2 & $\begin{array}{r}27,7 \\
(723)\end{array}$ \\
\hline Indéfinis & $\begin{array}{l}\% \\
N\end{array}$ & 10,4 & 10,5 & 11,1 & 9,2 & 8,5 & 9,4 & 10,1 & $\begin{array}{r}9,7 \\
(254)\end{array}$ \\
\hline TOTAL & $\%$ & $\begin{array}{c}4,4 \\
115\end{array}$ & $\begin{array}{r}9,1 \\
237\end{array}$ & $\begin{array}{l}13,1 \\
341\end{array}$ & $\begin{array}{l}24,1 \\
628\end{array}$ & $\begin{array}{l}13,9 \\
363\end{array}$ & $\begin{array}{l}19,6 \\
511\end{array}$ & $\begin{array}{l}15,9 \\
415\end{array}$ & $\begin{array}{c}100,0 \\
(2610)^{*}\end{array}$ \\
\hline
\end{tabular}

* Les arrangements de production sans destinataires sont exclus, soit 112 propositions relatives à la création, la modification ou l'abolition d'institutions. 
45,2 \% de ses interventions législatives à l'intention des travailleurs, s'explique, rappelons-le à nouveau, par la révision en profondeur des lois relatives aux corporations professionnelles. Si l'on ne tient pas compte de cette période un peu exceptionnelle, les gouvernements du PQ seraient à peine plus favorables aux travailleurs que ceux du Parti libéral. Les gouvernements du PLQ n'offrent généralement pas plus aux entrepreneurs que les autres gouvernements. Si l'on excepte la période du gouvernement libéral 62-66, où les entrepreneurs ont été la cible de $27 \%$ des interventions, le premier gouvernement du $P Q$ arrive bon deuxième dans sa production d'interventions bénéficiant aux entrepreneurs $(16,2 \%)$. La proportion des interventions qui s'adressent aux consommateurs évolue en dents de scie, indépendamment des partis au pouvoir.

Les bénéfices procurés à travers les interventions législatives de la mission sociale sont non monétaires plus de la moitié du temps $(52,8 \%)$. Il est impossible de déterminer, faute d'informations suffisantes, la nature des bénéfices offerts pour près de $27 \%$ des observations. Les bénéfices des interventions sont explicitement monétaires pour seulement $4,6 \%$ des cas alors qu'elles véhiculent des bénéfices monétaires non explicites dans $15,9 \%$ des mesures gouvernementales. Un bénéfice monétaire est considéré comme explicite s'il est décrit en référence à un montant d'argent alors qu'il est jugé non explicite s'il se limite à mentionner la distribution d'argent sans précision au sujet du montant. Les deux tiers des bénéfices monétaires non explicites s'adressent aux détenteurs de facteurs de production, les consommateurs devant se contenter $\mathrm{du}$ dernier tiers.

II importe maintenant de s'interroger sur le partage des coûts de ces interventions. Le tableau 7 montre que les mesures législatives ne comportent pas d'informations suffisantes sur l'imputation de coûts à des destinataires pour près de $70 \%$ des observations. Les travailleurs doivent supporter des coûts dans $13 \%$ des interventions de la mission sociale, comparativement à $11 \%$ pour les entrepreneurs, et à 4,6\% pour les consommateurs. Ces tendances lourdes ne sont jamais radicalement bouleversées par l'avènement de nouveaux partis politiques au pouvoir.

Par ailleurs, l'appariement des bénéfices aux coûts montre qu'il y a peu de transferts explicites de coûts d'une catégorie de particuliers à l'autre. Dans les cas où les coûts peuvent être mis en relation aux bénéfices, c'est-à-dire pour $31 \%$ des interventions, les entrepreneurs absorbent des coûts $62 \%$ du temps, cette proportion grimpant à $85 \%$ pour les consommateurs et à $87 \%$ pour les travailleurs. Les consommateurs absorbent le coût de bénéfices allant aux entrepreneurs à 3 occasions alors que les entrepreneurs en font autant pour les 
TABLEAU 7

Destinataires des coûts des interventions législatives, 1960-1985

\begin{tabular}{|c|c|c|c|c|c|c|c|c|c|}
\hline \multirow{2}{*}{\multicolumn{2}{|c|}{ Destinataires }} & \multicolumn{8}{|c|}{ Gouvernement } \\
\hline & & $\begin{array}{l}\text { PLQ } \\
60-62\end{array}$ & $\begin{array}{c}\text { PLQ } \\
62-66\end{array}$ & $\begin{array}{l}\text { UN } \\
66-70\end{array}$ & $\begin{array}{l}\text { PLQ } \\
\mathbf{7 0 - 7 3}\end{array}$ & $\begin{array}{l}\text { PLQ } \\
73-76\end{array}$ & $\begin{array}{c}P Q \\
76-81\end{array}$ & $\begin{array}{c}P Q \\
81-85\end{array}$ & $\begin{array}{l}\text { Total } \\
60-85\end{array}$ \\
\hline Entrepreneurs & $\%$ & 4,3 & 16,5 & 6,4 & 11,1 & 10,2 & 15,1 & 8,9 & $\begin{array}{l}11,0 \\
(287)\end{array}$ \\
\hline Travaiileurs & $\%$ & 11,3 & $10, \overline{1}$ & $7, \overline{9}$ & 22,4 & $\overline{8}, \overline{5}$ & 9,4 & 14,2 & $\begin{array}{r}13,1 \\
(343)\end{array}$ \\
\hline $\begin{array}{l}\text { Autres } \\
\text { producteurs }\end{array}$ & $\%$ & 2,6 & 0,0 & 3,8 & 2,7 & 5,2 & 1,8 & 2,2 & $\begin{array}{l}2,7 \\
(70)\end{array}$ \\
\hline Consommateurs & $\%$ & 6,1 & 7,6 & 5,3 & 3,5 & 5,5 & 4,9 & 2,4 & $\begin{array}{r}4,6 \\
(120)\end{array}$ \\
\hline Indéfinis & $\%$ & 75,6 & 65,8 & 76,5 & 60,2 & 70,5 & 68,8 & 72,3 & $\begin{array}{r}68,5 \\
(1789)\end{array}$ \\
\hline TOTAL & $\begin{array}{l}\% \\
N\end{array}$ & $\begin{array}{l}4,4 \\
115\end{array}$ & $\begin{array}{r}9,1 \\
237\end{array}$ & $\begin{array}{l}13,1 \\
341\end{array}$ & $\begin{array}{l}24,1 \\
628\end{array}$ & $\begin{array}{l}13,9 \\
363\end{array}$ & $\begin{array}{l}19,6 \\
512\end{array}$ & $\begin{array}{l}15,9 \\
415\end{array}$ & $\begin{array}{c}100,0 \\
(2610)^{*}\end{array}$ \\
\hline
\end{tabular}

* Les arrangements de production sans destinataires sont exclus. 
consommateurs à 31 reprises. Les travailleurs absorbent dans 3 interventions les coûts des bénéfices qui leur sont destinés alors qu'ils assument 30 fois les coûts des bénéfices réservés aux consommateurs. On peut cependant penser qu'il y a transfert implicite de richesse à l'avantage des travailleurs étant donné que ceux-ci jouissent plus souvent que les autres de bénéfices dont les coûts sont indéfinis (704 interventions). Les consommateurs reçoivent des bénéfices à coûts indéfinis à 541 reprises alors qu'il en va de même pour les entrepreneurs dans 146 interventions. La législation de la mission sociale n'est donc pas aussi régressive que le prédit la théorie des choix collectifs qui, rappelons-le, affirme que les bénéfices vont aux détenteurs de facteurs de production alors que les coûts sont diffusés à l'ensemble des consommateurs et des contribuables.

\section{Une approche modifiée de la mission sociale}

Comme les détenteurs de facteurs de production constituent la cible de près des trois quarts des propositions de la mission sociale, il importe de la réexaminer en y soustrayant deux domaines d'intervention qui pourraient tout autant être inclus dans la mission économique de l'État; ce sont les domaines des relations de travail et des conditions de travail. Cette modification est lourde de conséquences. Les destinataires visés par les interventions concernant le travail sont, en effet, essentiellement des détenteurs de facteurs de production. Cet état de fait crée donc un biais et gonfle indûment le nombre $d^{\prime}$ interventions de la mission sociale qui concernent les détenteurs de facteurs de production.

L'exclusion des interventions relatives aux relations et aux conditions de travail diminue le nombre d'interventions de 2722 à 1337. De ce nombre, $49,1 \%$ sont destinées aux consommateurs, $33,2 \%$ aux détenteurs de facteurs de production et $17,6 \%$ ont des cibles de type indéfini. (Tableau 8).

Cette nouvelle définition de la mission sociale affecte aussi la répartition des coûts. Le retrait des domaines conditions et relations de travail diminue fortement les coûts absorbés par les travailleurs qui chutent ainsi de $9 \%$ à 2,2 \%. La proportion des coûts assumés par les entrepreneurs demeure à peu près inchangée alors que les consommateurs voient leurs coûts grimper de $4,6 \%$ à $8,5 \%$. En bref, moins du quart des interventions sont explicitement liées à des coûts $(-6 \%)$. Finalement, cette modification de la mission sociale fait aussi disparaître le transfert de la richesse à l'endroit des travailleurs au profit des consommateurs, renforçant ainsi notre conclusion au sujet de la «non régressivité » des mesures législatives de la mission sociale. 


\section{TABLEAU 8}

\section{Comparaison des destinataires des bénéfices de la mission sociale après modification}

\begin{tabular}{|lccc|}
\hline Destinataires & $\begin{array}{c}\text { Mission } \\
\text { sociale }\end{array}$ & $\begin{array}{c}\text { Mission } \\
\text { sociale } \\
\text { modifiée }\end{array}$ & Écarts \\
\hline Entrepreneurs & 338 & 146 & -192 \\
& $13,0 \%$ & $10,9 \%$ & $-2,0 \%$ \\
Travailleurs & 1080 & 173 & -907 \\
& $41,3 \%$ & $12,9 \%$ & $-28,4 \%$ \\
Autres & 219 & 126 & -93 \\
& $8,4 \%$ & $9,4 \%$ & $+1,0 \%$ \\
Consommateurs & 723 & 656 & -67 \\
& $27,7 \%$ & $49,1 \%$ & $+21,4 \%$ \\
Indéfinis & 254 & 236 & -18 \\
& $9,7 \%$ & $17,6 \%$ & $+7,9 \%$ \\
\hline
\end{tabular}

\section{Conclusion}

Le sens commun incite à penser que les interventions législatives de la mission sociale bénéficient massivement aux consommateurs. Les résultats de l'analyse des lois sont toutefois très sensibles à la définition du concept de mission sociale. Ainsi, les consommateurs sont les bénéficiaires de près de $30 \%$ des interventions législatives alors que les détenteurs de facteurs de production constituent la cible d'un peu plus de $60 \%$ des bénéfices des mesures législatives lorsque la mission sociale incorpore les domaines des conditions et des relations de travail. Inversement, l'exclusion de ces deux domaines d'intervention montre que les consommateurs deviennent les bénéficiaires de près de $50 \%$ des interventions législatives alors que les détenteurs de facteurs de production sont la cible du tiers des mesures dont près de $13 \%$ réservé aux travailleurs.

Bien que les coûts de près de $70 \%$ des bénéfices des mesures législatives soient indéfinis, il est possible de conclure qu'il y a transfert implicite de richesse des producteurs vers les consom- 
mateurs puisque ces derniers jouissent plus souvent que les autres groupes de particuliers de bénéfices dont les coûts sont indéfinis. La législation de la mission sociale ne s'avère donc pas aussi régressive que le prédit la théorie déductive du transfert de richesse en état $\mathrm{d}^{\prime}$ incertitude. Ces tendances lourdes ne sont pas radicalement affectées par les changements de partis au pouvoir, que l'on soit dans la perspective de la mission sociale modifiée ou non : les gouvernements du $P Q$ favorisent en effet à peine plus les travailleurs que ceux du PLQ; le PLQ n'est pas beaucoup plus offrant à l'endroit des entrepreneurs que le $P Q$; finalement, les consommateurs tendent à recevoir un peu plus de bénéfices du PLQ que du PQ. Au total, force est de conclure que la simple connaissance des étiquettes idéologiques des partis ne permet pas de prédire les bénéficiaires des biais de la législation de la mission sociale. Les partis gouvernementaux constituent des réalités plus complexes qu'on veut souvent le croire.

Cette complexité tient d'abord au fait qu'un parti nouvellement porté au pouvoir hérite du réservoir des mesures législatives adoptées par les gouvernements précédents. Les engagements hérités des législatures précédentes ne peuvent pas facilement être répudiés même si, en théorie, un parti qui arrive au pouvoir peut abroger toutes les lois de ses prédécesseurs. Les engagements enregistrés dans les lois représentent en fin de compte une formidable force $d^{\prime}$ inertie qui incite les partis au pouvoir à accepter tacitement les lois votées par leurs prédécesseurs. L'amplitude de cette force d'inertie pourrait expliquer l'absence de variations importantes pour les bénéficiaires des interventions législatives lors des changements de partis au pouvoir.

On peut aussi conclure que les mesures législatives de la mission sociale entretiennent le mythe de la gratuité : les coûts et les bénéfices sont identifiés simultanément dans moins du tiers des interventions. On peut arguer que nos institutions politiques légitimisent cette disjonction pour les mesures gouvernementales qui véhiculent des bénéfices monétaires car alors, les ministres qui dispensent les bénéfices sociaux ne sont généralement pas ceux qui ont le mandat de fixer les taxes et les impôts. Cet argument ne résiste toutefois pas à l'épreuve des faits puisqu'à peine le cinquième des bénéfices de la législation de la mission sociale comporte des bénéfices à caractère monétaire. Cette propension des partis gouvernementaux à dissimuler les coûts de leurs interventions législatives produit implicitement des transferts de richesse : $90 \%$ des mesures législatives de la mission sociale produisent des bénéfices dont la consommation est réservée à des groupes particuliers. En fin de compte, la législation de la mission sociale produit massivement des 
biens de clubs dont les coûts sont indéfinis, mais dont la consommation est réservée à des catégories particulières d'individus. La régressivité de la législation de la mission sociale prend donc appui sur la dissimulation des coûts des bénéfices résultant des interventions gouvernementales.

La méthode développée pour l'analyse des interventions législatives de la mission sociale pourrait facilement être reprise dans le but de saisir la portée redistributive des interventions des trois autres missions de l'État, l'administration et le gouvernement, l'éducation et la culture, puis finalement, l'économie. La propension à dissimuler les coûts des mesures gouvernementales ainsi que la tendance à intervenir en effectuant des transferts de ressources entre les groupes ne sont pas propres aux interventions gouvernementales de la mission sociale. Ainsi, l'analyse des lois de la mission économique pourrait laisser voir un modèle de transfert implicite de richesse allant des consommateurs et des contribuables vers les détenteurs de facteurs de production.

\section{Note}

* Ce projet a été réalisé grâce à une subvention du Conseil québécois de la recherche sociale. Les auteurs remercient François Michaud pour son excellent travail concernant l'exploitation informatique des données.

\section{Références}

DAHLBY, B.G., 1986, " L'incidence des dépenses et des impôts au Canada : un tour d'horizon ": 125-172, dans F. Vaillancourt, éd., La répartition du revenu et de la sécurité économique au Canada, Ministère des Approvisionnements et services du Canada.

Downs, A., 1957, An Economic Theory of Democracy, New York, Harper and Row.

FreY, B.S., 1983, Democratic Economic Policy: A Theoretical Introduction, Oxford, Martin Robertson.

Guindon, H., 1964, "Social Unrest, Social Class, and Quebec Bureaucratic Revolution ", Queen's Quarterly, 1971, 15 août 1962.

HAYEs, M., 1981, Lobbyists and Legislators : A Theory of Political Markets, New Brunswick, Rutgers University Press.

HAYES, M.T., 1978, "The semi-Sovereign Pressure Groups : A Critique of Current Theory and an Alternative Typology ", Journal of Politics, 40 : 134161.

Lemelin, C., 1980, La répartition du coût des études universitaires, Conseil des universités, Gouvernement du Québec. 
McCormick, R.E. et R.D. Tollison, 1981, Politicians, Legislation and the Economy. An Inquiry into the Interest-Group Theory of Government, Boston, Martinus Nijhoff Publishing.

Mcroberts, K., 1988, Quebec Social Change and Political Crisis, Toronto, MClelland et Stewart, $3^{\mathrm{e}}$ édition.

MOE, T.M., 1980, The Organization of Interests. Incentives and the Internal Dynamics of Political Interest Groups, Chicago, The University of Chicago Press.

MOE, T.M., 1981, "Toward a Broader View of Interest Groups ", The Journal of Politics, 43-2 : 531-543.

Mueller, D.C., 1987, "The Growth of Government: A Public Choice Perspective ", International Monetary Fund, Staff papers, 34-1 : 115-149.

MONTMARQUeTte, C., 1988, Le marché politique : Qu'est-ce qui est produit ? Qui y participe? Qui en profite?, Cahier 1488, Département d'économique et C.R.D.E., Université de Montréal, 32 pages.

Otson, M., Jr., 1965, The Logic of Collective Action. Public Goods and the Theory of Groups, New York, Schoken Books.

Otson, M., Jr.,1982, The Rise and Decline of Nations, Economic Growth, Stagflation and Social Rigidities, New Haven, Yale University, paper edition.

Payette, M. et F. Vaillancourt, 1986, "L'incidence des recettes et des dépenses gouvernementales au Québec en 1981 ", L'actualité économique, 62-3 : 409-441.

Rose, R., 1983, Understanding Big Government. A Program Approach, Beverly Hills, Ca., Sage Publications.

Thurow, L.C., 1980, The Zero Sum Society, New York, Basic Books.

Tullock, G., 1983, Economics of Income Redistribution, Boston, Kliewer-Nijoff. 